\title{
Effects of antibody to receptor activator of nuclear factor K-B ligand on inflammation and cartilage degradation in collagen antibody-induced arthritis in mice
}

Sakie Funato ${ }^{1,4}$, Akihiro Matsunaga ${ }^{1,2}$, Koei Oh $^{2,3}$, Yoichi Miyamoto $^{1 *}$, Kentaro Yoshimura ${ }^{1}$, Junichi Tanaka ${ }^{5}$, Dai Suzuki ${ }^{1}$, Risa Uyama', Hiroaki Suzuki ${ }^{1}$, Kenji Mishima ${ }^{5}$, Masanori Nakamura ${ }^{3}$, Osamu Namiki ${ }^{2}$ Kazuyoshi Baba ${ }^{4}$, Katsunori Inagaki ${ }^{2}$ and Ryutaro Kamijo ${ }^{1}$

\begin{abstract}
Background: Rheumatoid arthritis (RA) is an inflammatory disease that leads to destruction of both articular cartilage and bone tissues. In rheumatic joints, synoviocytes and T-lymphocytes as well as bone cells produce the receptor activator of nuclear factor K-B (RANK) ligand (RANKL), which binds to RANK on the surface of osteoclasts and their precursor cells to induce differentiation and activation of osteoclasts. Hence, inhibition of RANKL may be a promising approach to suppress osteolysis in RA. On the other hand, RANKL production by lymphocytes indicates the possibility that its inhibition would be effective to suppress inflammation in RA. In addition, it has been reported that cathepsin $\mathrm{K}$, a predominant cysteine protease in osteoclasts, is involved in cartilage destruction in RA model mice. Here, we evaluated the effects of an anti-RANKL antibody on inflammation in footpads and degradation of articular cartilage in RA model mice.
\end{abstract}

Results: We induced arthritis in mice by injection of anti-type II collagen antibodies and lipopolysaccharide (LPS). Inhibition of RANKL by an anti-RANKL antibody (OYC1, Oriental Yeast, Tokyo, Japan) was confirmed by increased bone volume in the metaphysis of tibias. Swelling in either limb until day 14 was seen in 5 of 6 mice injected with anti-collagen antibodies and LPS without treatment with OYC1, while that was seen in 4 of 5 mice treated with OYC1. The average arthritis scores on day 14 in those groups were 2.17 and 3.00, respectively, indicating that OYC1 did not ameliorate inflammation in the limbs. Histological analyses indicated that OYC1 does not protect articular cartilage from destruction in mice with arthritis.

Conclusions: Our present study failed to show the effectiveness of an anti-RANKL antibody to ameliorate inflammation in the limbs or protect articular cartilage from degradation in a collagen antibody-induced arthritis mouse model.

Keywords: Rheumatoid arthritis, Collagen antibody-induced arthritis, RANKL, Antibody, Inflammation, Articular cartilage

\section{Background}

Rheumatoid arthritis (RA) is an inflammatory disease that leads to destruction of both articular cartilage and bone tissues. An articular cavity is a space enclosed by articular cartilage and synovial membranes. Single-layered cells compose a synovial membrane in normal joints, while in RA they

\footnotetext{
* Correspondence: yoichim@dent.showa-u.ac.jp

'Department of Biochemistry, Showa University School of Dentistry, 1-5-8

Hatanodai, Shinagawa, Tokyo 142-8555, Japan

Full list of author information is available at the end of the article
}

proliferate in inflammatory conditions to form a pannus that destroys both cartilage and bone tissues [1]. It is well known that osteoblasts and/or osteocytes stimulated by physiological bone-resorbing factors such as activated vitamin D and parathyroid hormone produce the receptor activator of nuclear factor $\mathrm{K}-\mathrm{B}$ (RANK) ligand (RANKL), which binds to RANK distributed on the plasma membrane of osteoclasts and their precursor monocytes/macrophages. Interaction between RANKL and RANK induces differentiation and activation of osteoclasts [2,3]. Inflammatory cytokines 
including tumor necrosis factor- $\alpha$, interleukin-1, interleukin6 , and interleukin-17 induce the expression of RANKL in osteoblasts, which augments bone destruction by osteoclasts in inflammatory conditions [4]. In addition, synovial fibroblasts and activated $\mathrm{T}$ lymphocytes abundantly produce RANKL, which is considered to contribute to osteoclastogenesis in RA [5]. Therefore, inhibition of the interaction between RANKL and RANK may be a promising approach to suppress osteolysis in RA. Denosumab, a fully human monoclonal antibody against RANKL, has been used clinically for treatment of osteoporosis and bone erosion associated with multiple myeloma and bone metastasis from tumors [6,7]. In addition, clinical studies on the effectiveness of denosumab against RA revealed that denosumab suppressed bone erosion [8].

The RANKL/RANK system functions not only in bones, but also in various tissues and cells including the immune system, vascular system, skin, and central nervous system [9]. The existence of this system in the immune system suggests that inhibition of RANKL can suppress the onset of RA and consequently inhibit cartilage degradation. It has been also reported that cathepsin $\mathrm{K}$, a predominant cysteine protease in osteoclasts, is expressed in osteoclasts in contact with articular cartilage in RA patients and stabilized by glycosaminoglycans, such as chondroitin sulfate, which are abundantly produced by chondrocytes $[10,11]$. It was also reported that inhibition of cathepsin $\mathrm{K}$ suppressed cartilage degradation in collagen-induced arthritis in mice [12]. In addition, a clinaical study regarding the effects of denosumab on RA showed a transient decrease in the blood level of C-terminal telopeptide of type II collagen [8]. These observations suggest that anti-RANKL antibodies may have potential to inhibit degradation of articular cartilage associated with RA.

In the present study, we evaluated the effects of an anti-RANKL antibody on inflammation in footpads and degradation of articular cartilage in collagen antibodyinduced arthritis model mice.

\section{Results \\ Induction of arthritis and validation of anti-RANKL antibody in mice}

We examined the effects of an anti-RANKL antibody on inflammation in the joints and degradation of articular cartilage using a collagen antibody-induced arthritis mouse model. Male DBA1/J mice were divided into 4 experimental groups (6 in each), i.e., RA-/Ab-, RA-/Ab+, RA+/Ab-, and $\mathrm{RA}+/ \mathrm{Ab}+$ (Figure 1). RA+ mice were injected with a cocktail of antibodies to type II collagen and lipopolysaccharide (LPS) (Chondrex, Inc., Redmond, WA) to induce arthritis. RA- mice were the control without injection of the collagen antibodies and LPS. Ab+indicate that mice belonging to these groups were treated with the anti-RANKL antibody (OYC1, Oriental Yeast, Tokyo, Japan). Ab- mice are the control without treatment with the anti-RANKL antibody. Unfortunately, 1 of the $6 \mathrm{RA}+/ \mathrm{Ab}+$ mice died within 1 day after subcutaneous injection of the anti-RANKL antibody from peritonitis possibly caused by an inappropriate intraperitoneal injection of the anesthetic agent.

$\mu \mathrm{CT}$ analyses indicated that administration of OYC1 antiRANKL antibody increased bone mass both in tibias from $\mathrm{RA}$ - and RA+ mice (Figure 2A). The bone volume fraction (BV/TV) of trabecular bone in tibias from mice injected with the OYC1 anti-RANKL antibody (RA-/Ab+ mice) was significantly greater as compared to that in RA-/Ab- mice (Figure 2B). The same effect of the antibody was observed in RA+ mice (Figure 2C). Trabecular thickness (Tb.Th) was not affected by OYC-1 anti-RANKL antibody in either RAor RA+ group (Figure 2D, E). While OYC1 antibody did not change trabecular number (Tb.N) in RA- mice (Figure $2 \mathrm{~F}$ ), the antibody significantly increased Tb.N in RA+ mice (Figure 2G). While trabecular space (Tb.Sp) tended to decline in mice received the anti-RANKL antibody in both $\mathrm{RA}$ - and RA+ groups, the effect of the antibody on Tb.Sp was not significant (Figure $2 \mathrm{H}, \mathrm{I}$ ). These quantitative $\mu \mathrm{CT}$ analyses indicated that the amount of OYC1 anti-RANKL antibody administered (5 $\mathrm{mg} / \mathrm{kg}$ ) was sufficient for inhibition of RANKL in our experimental model, as previously reported [13].

\section{Ineffectiveness of anti-RANKL antibody on inflammation induced by anti-collagen antibodies}

We measured the footpad thickness of 4 limbs daily after injection of a cocktail of antibodies to type II collagen. Additional file 1: Figure S1 shows change in the thickness of each paw of the individual mice. Thickness of footpads was hardly changed in RA- mice in either $\mathrm{Ab}$ - or $\mathrm{Ab}+$ group (Additional file 1: Figure S1A, C, E, G). On the other hand, some of the mice injected with a cocktail of antibodies to type II collagen on day 0 and LPS on day 3 showed swelling in the paws, especially those in fore limbs of both $\mathrm{Ab}$ - and $\mathrm{Ab}+$ mice (Additional file 1: Figure S1B, D). Change in the thickness of hind paws was small (Additional file 1: Figure S1F, H). Then we summarized the frequency of swelling in each paw of the individual mouse observed 14 days after injection of anti-type II collagen antibodies (Table 1). Swelling frequency in either limb until day 14 in the $\mathrm{RA}+/ \mathrm{Ab}$ - group occurred in 5 of the 6 mice, whereas that was seen in 4 of 5 in the $R A+/ A b+$ group.

We also determined the arthritis score of mice everyday as an index of severity of inflammation based on the frequency and extent of swelling and reddening in the limbs. The RA score for RA- mice did not increase irrespective of whether they were given anti-RANKL antibody $(\mathrm{RA}-/ \mathrm{Ab}+)$ or not (RA-/Ab-). On the other hand, the average arthritis score for the $\mathrm{RA}+/ \mathrm{Ab}$ - mice on day 12 was $2.17 \pm 1.33$ (mean $\pm \mathrm{SD}$ ), while that for the RA $+/ \mathrm{Ab}+$ mice was $3.00 \pm 0$ (Figure 3 ). These results show 


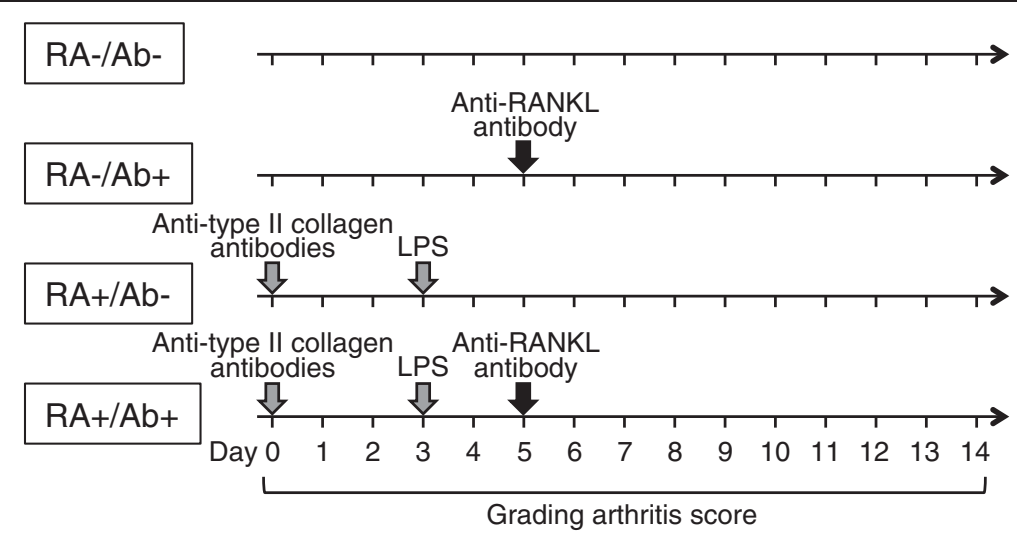

Mice on day 14: Limbs $\longrightarrow$ Histological analysis

Figure 1 Experimental design. Eight-week-old male DBA1/J mice were divided into 4 groups (6 in each), i.e., RA-/Ab-, RA-/Ab+, RA+/Ab-, and RA+/Ab+. A cocktail of anti-type II collagen antibodies and E. coli LPS was injected intra-peritoneally into the RA+ mice on days 0 and 3 , respectively. RA- mice were the control without injection of the anti-type II collagen antibodies and LPS. The OYC1 anti-RANKL monoclonal antibody ( $5 \mathrm{mg} / \mathrm{kg}$ ) was injected subcutaneously into the Ab+ mice on day 5 . Ab- mice were the control without injection the anti-RANKL antibody. Arthritis scores were determined daily from day 0 to 14. The 4 limbs were removed on day 14 for analysis of histological changes in the joints.

that administration of the anti-RANKL antibody did not have an effect to suppress inflammation induced by antibodies against type II collagen and LPS.

Representative histological images of limb joints are shown in Figure 4. Synovial thickening, one of the typical changes seen in joints affected by RA, was observed in joints from both the RA+/Ab- (Figure 4C, G, K, O) and RA+/Ab+ (Figure 4D, H, L, P) mice. No significant difference was seen in regard to synovial thickening or infiltration of inflammatory cells in the $\mathrm{RA}+/ \mathrm{Ab}$ - mice and $\mathrm{RA}+/ \mathrm{Ab}+$ mice, indicating that synovitis was not ameliorated by administration of the anti-RANKL antibody.

Ineffectiveness of anti-RANKL antibody on degradation of cartilage induced by anti-collagen antibodies

Representative magnified images of articular cartilage from forelimbs of the RA-/Ab-, RA-/Ab+, RA+/Ab-, and $\mathrm{RA}+/ \mathrm{Ab}+$ mice are shown in Figures 5A-D. The articular cartilage surface in the RA-/Ab- and RA-/Ab+ mice had a smooth and clean appearance (Figure 5A, B), whereas erosion was evident in that from $\mathrm{RA}+$ mice irrespective of anti-RANKL antibody administration (Figure 5C, D). Cartilage matrix stained with Safranin O was reduced in $\mathrm{RA}+$ mice (Figure $5 \mathrm{C}, \mathrm{D}$ ) in comparison with that in RA- mice (Figure 5A, B). Anti-RANKL antibody did not ameliorate the loss of cartilage matrix in $\mathrm{RA}+$ mice (Figure 5C, D). These findings indicated that the OYC1 anti-RANKL antibody did not provide a protective effect on cartilage in the present collagen antibodyinduced arthritis model.

\section{Discussion}

Denosumab, a fully human monoclonal antibody against RANKL, is used clinically for treatment of osteoporosis and bone erosion associated with multiple myelomas and bone metastasis from those tumors [6,7]. In addition, the effectiveness of denosumab for treatment of bone erosion in RA is promising. On the other hand, degradation of articular cartilage is another serious problem encountered in patients with RA. Since it is known that RANKL participates in immunological reactions [14] and cathepsin $\mathrm{K}$, one of the major proteases secreted by osteoclasts, is involved in cartilage degradation [12], it is important to determine if inhibition of RANKL can ameliorate cartilage degradation in RA. In the present study, we examined the effects of an anti-RANKL antibody on inflammation and cartilage degradation in a mouse model of RA induced by injection of a cocktail of antibodies to type II collagen.

We employed an RA model in mice produced by injection of antibodies against type II collagen and LPS to evaluate effectiveness of treatment with anti-RANKL antibody. Swelling of the paws (Table 1 and Additional file 1: Figure S1), increment in the RA score (Figure 3), and accumulation of inflammatory cells in the joints (Figure 4) indicated that inflammation was induced in the limbs by the treatments with antibodies against type II collagen and LPS. Reduced staining with Safranin O and the rough surface of the articular cartilage also suggested the degradation of the cartilage matrix in the joints in RA+ mice (Figure 5). Hence it is considered to be appropriate to use this model for evaluation of the 


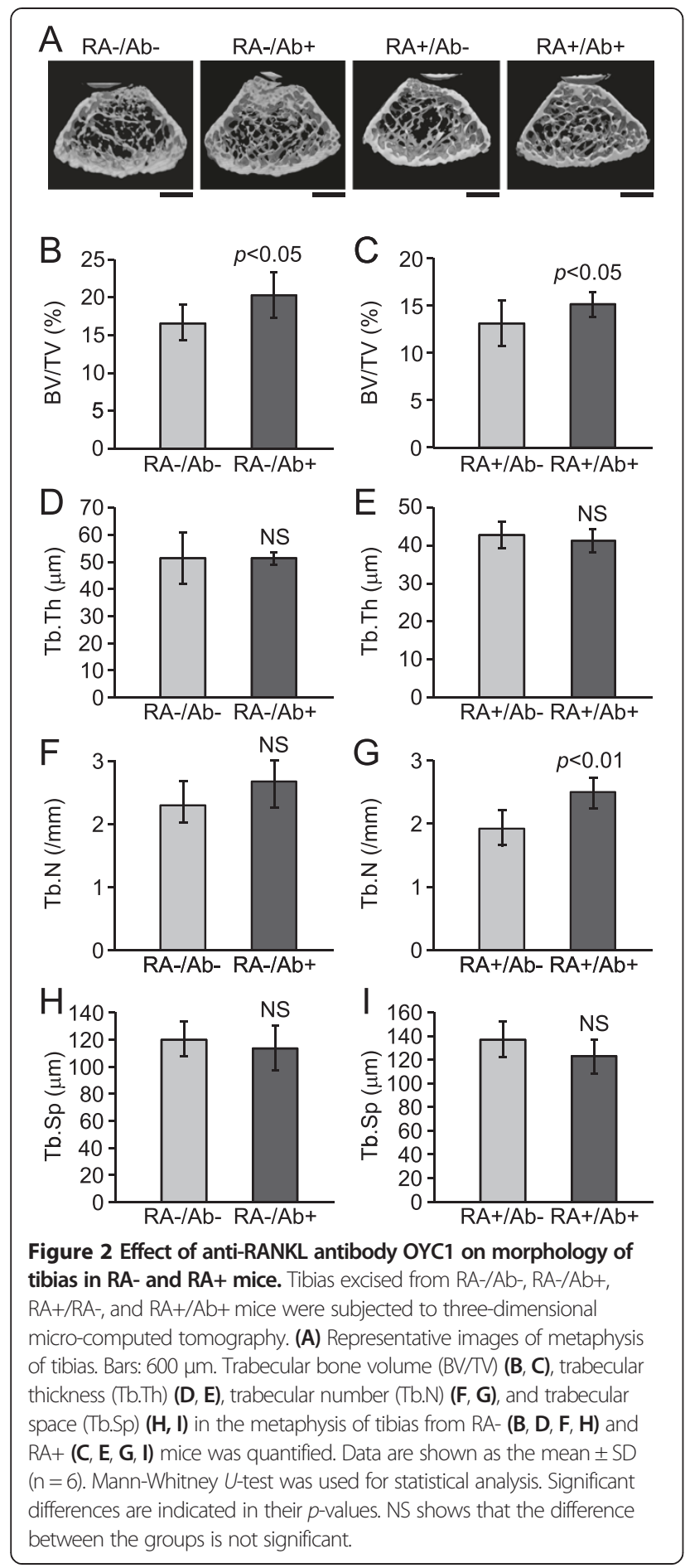

effect of anti-RANKL antibody on inflammation and cartilage degeneration in RA.

It was previously reported that the OYC1 anti-RANKL antibody was effective to suppress osteoclast formation in mice from 1 to at least 28 days after subcutaneous injection at a dose of $5 \mathrm{mg} / \mathrm{kg}$ of body weight [13]. In the present study, the value for BV/TV in the metaphysis of
Table 1 Frequency of swelling in limb pads

\begin{tabular}{|c|c|c|c|c|c|}
\hline \multirow[t]{2}{*}{ Group } & \multicolumn{2}{|c|}{ Fore limb } & \multicolumn{2}{|c|}{ Hind limb } & \multirow[t]{2}{*}{ Total } \\
\hline & Left & $\overline{\text { Right }}$ & Left & $\overline{\text { Right }}$ & \\
\hline $\mathrm{RA}-/ \mathrm{Ab}-$ & $0 / 6$ & $0 / 6$ & $0 / 6$ & $0 / 6$ & $0 / 6$ \\
\hline $\mathrm{RA}-/ \mathrm{Ab}+$ & $0 / 6$ & $0 / 6$ & $0 / 6$ & $0 / 6$ & $0 / 6$ \\
\hline $\mathrm{RA}+/ \mathrm{Ab}-$ & $1 / 6$ & $5 / 6$ & $1 / 6$ & $0 / 6$ & $5 / 6$ \\
\hline $\mathrm{RA}+/ \mathrm{Ab}+$ & $4 / 5$ & $3 / 5$ & $1 / 5$ & $1 / 5$ & $4 / 5$ \\
\hline
\end{tabular}

Development of inflammation in the 4 limbs was evaluated daily by measuring the thickness of each footpad (Additional file 1: Figure S1). Occurrence of swelling in the limbs until day 14 is shown as a fraction, with the numerator and denominator representing the number of mice with swollen limb(s) and total number of mice in the group, respectively.

tibias was significantly increased by subcutaneous injection of the same dose of the OYC1 anti-RANKL antibody (Figure 2B, C), demonstrating that the activity of RANKL was also suppressed by OYC1 in our experimental model. Hence, the inability of this antibody to reduce inflammation in synovial membranes and degradation of articular cartilage is not due to insufficient inhibition of RANKL. Osteoprotegerin (OPG), a soluble decoy receptor for RANKL, is secreted by osteoblasts and other types of cells and inhibits interaction between RANKL and RANK by binding to the former $[15,16]$. There are several reports of the effects of OPG administration on the pathogenesis of RA in animal models [17-20], including findings that bone erosion and osteoclast functions were down-regulated by treatment with OPG, whereas that did not have a significant effect on inflammation or cartilage erosion [20]. Those reports and the present results indicate that the contribution

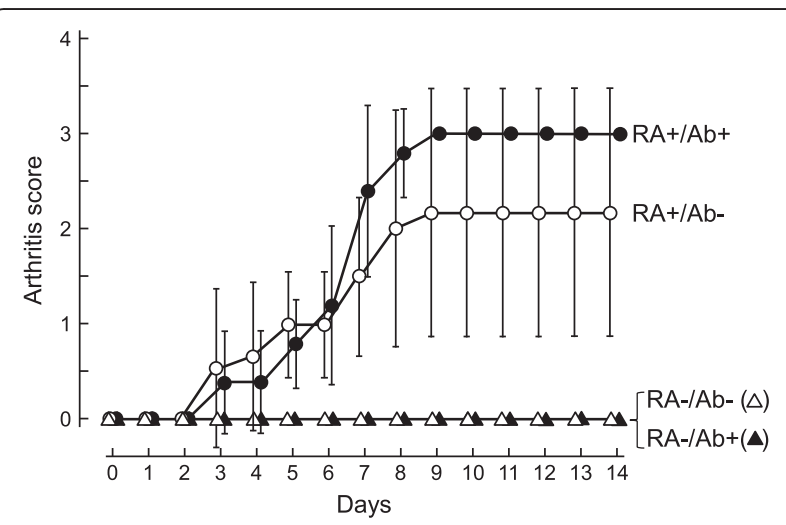

Figure $\mathbf{3}$ Change in arthritis score after injection of antibodies to type II collagen. Arthritis scores were determined daily after injection of a cocktail of antibodies to type II collagen according to the following criteria: 0 , normal; 1 , mild but definite redness and swelling of the ankle or wrist, or apparent redness and swelling limited to individual digits regardless of the number of affected digits; 2, moderate redness and swelling of ankle and wrist; 3 , severe redness and swelling of the entire paw including digits; 4 , maximally inflamed limb with involvement of multiple joints. Data are expressed as the mean $\pm \mathrm{SD}$. There was no statistical difference between $\mathrm{RA}+/ \mathrm{Ab}$ - group and RA+/Ab+ group (Steel-Dwass test). 


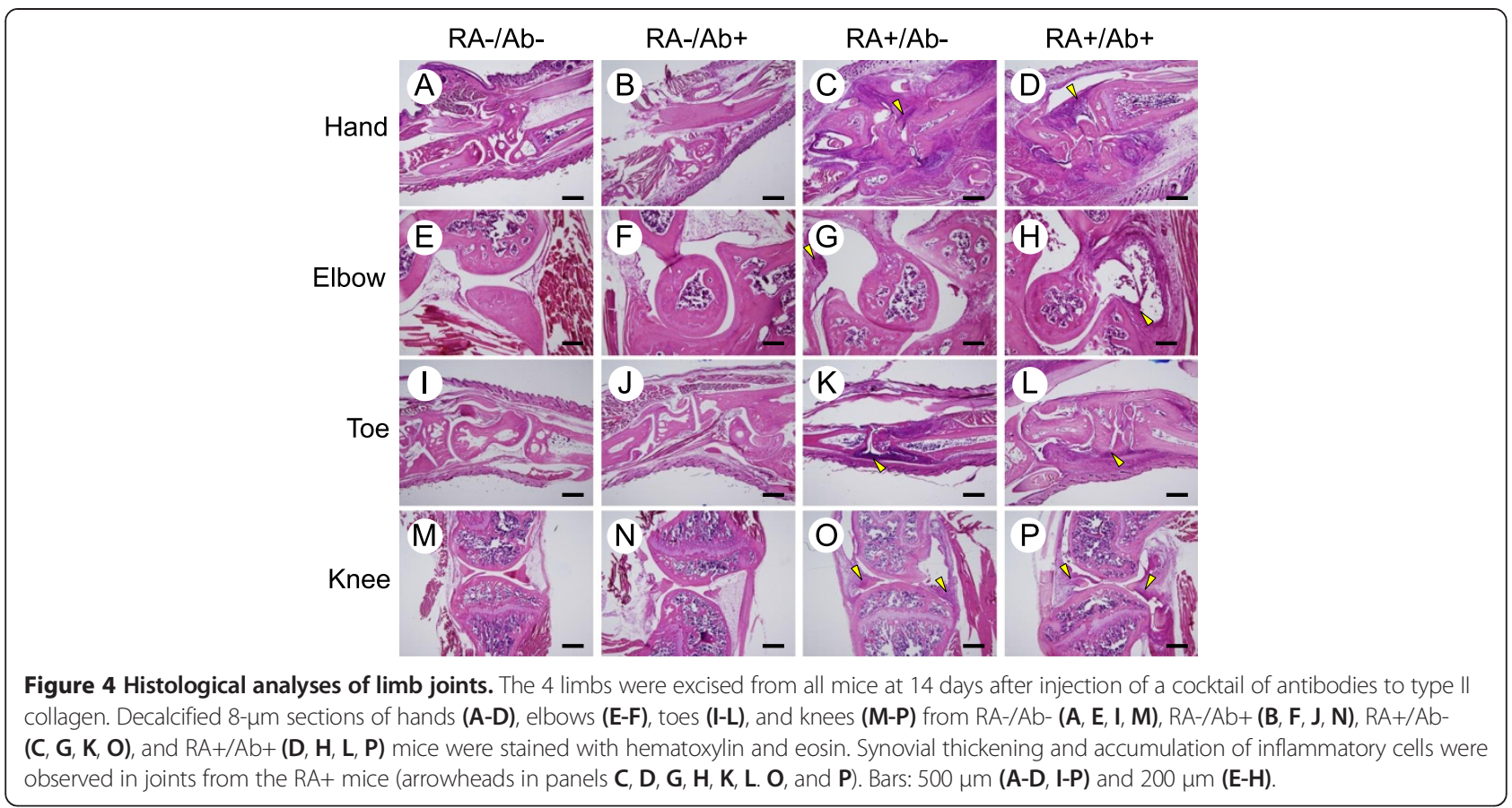

of RANKL to inflammation and cartilage erosion in RA is small.

Although human studies are required for evaluation of the effects of denosumab on cartilage erosion, the present animal study findings suggest that inhibition of RANKL by its antibodies is not effective for protection of articular cartilage from degradation associated with RA.

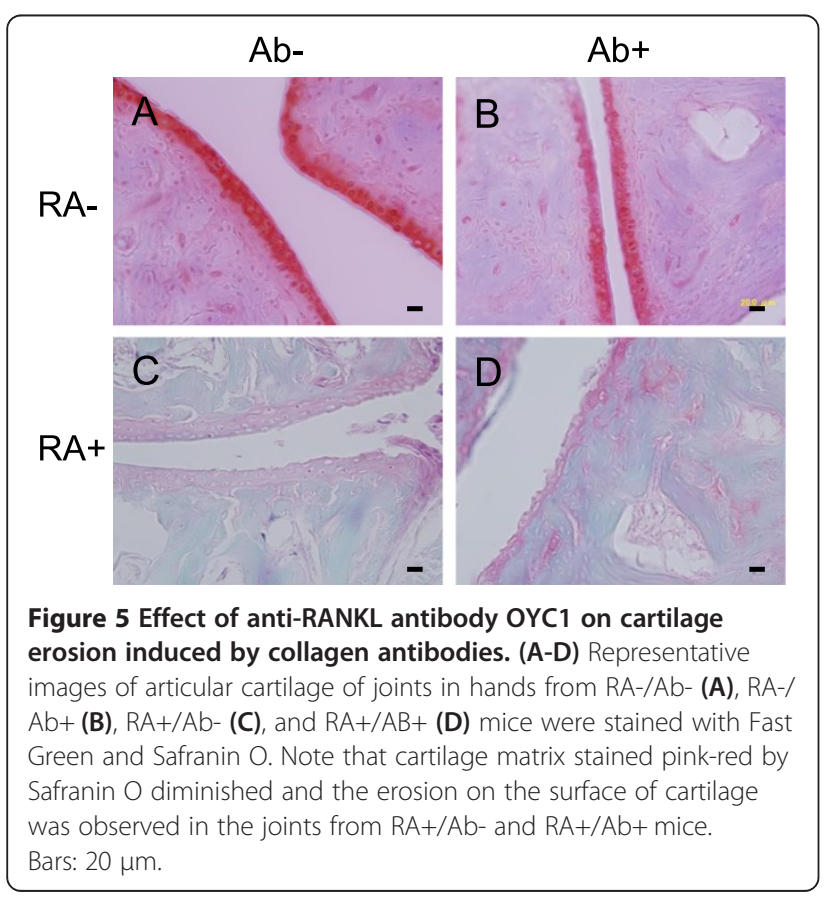

\section{Conclusion}

The anti-RANKL antibody OYC1 did not protect articular cartilage from degradation in collagen antibodyinduced arthritis model mice, despite inhibition of RANKL by the antibody shown by increased tibia bone volume.

\section{Materials and methods Reagents}

An Arthrogen-CIA ${ }^{\oplus}$ 5-clone cocktail kit with LPS from Escherichia coli O111:B4 (Chondrex) was used for induction of collagen antibody-induced arthritis in mice. The anti-mouse RANKL monoclonal antibody (OYC1) was obtained from Oriental Yeast Co., Ltd. An animal COMP ELISA kit was purchased from AnaMar AB (Göteborg, Sweden). All other chemicals and reagents were obtained from commercial sources.

\section{Mice and experimental groups}

Eight-week-old male DBA1/J mice (Japan SLC, Inc., Shizuoka, Japan) were randomly divided into 4 groups (6 in each), i.e., RA-/Ab-, RA-/Ab+, RA+/Ab-, and $\mathrm{RA}+/ \mathrm{Ab}+($ Table 1$)$. Mice in the $\mathrm{RA}+$ groups were given an intra-peritoneal injection of a cocktail of 5 clones of mouse monoclonal anti-type II collagen antibodies (1.5 mg/0.15 mL/head) on day 0 , followed by an intraperitoneal injection of $E$. coli LPS $(50 \mu \mathrm{g} / 0.1 \mathrm{~mL} / \mathrm{head})$ on day 3 according to the manufacturer's instruction (Chondrex). RA- mice were the control without injection of the anti-type II collagen antibodies and LPS. The OYC1 
anti-RANKL monoclonal antibody ( $5 \mathrm{mg} / \mathrm{kg}$ body weight) was injected subcutaneously into mice in the $\mathrm{Ab}+$ groups on day 5 , while mice in the Ab- groups were not given that treatment. All mice were housed in a specific pathogen-free environment, and given free access to food and water. All experiments performed in this study were approved by the Ethical Board for Animal Experiments of Showa University, Tokyo, Japan (approval number: 14011).

\section{Scoring of RA}

We observed mice daily for development of inflammation in the 4 limbs from day 0 to 14 . The thickness of each footpad was measured using a slide caliper. In addition, the severity of inflammation was scored according to the criteria of Chondrex, as follows: 0 , normal; 1 , mild but definite redness and swelling of the ankle or wrist, or apparent redness and swelling limited to individual digits regardless of the number of affected digits; 2, moderate redness and swelling of ankle and wrist; 3 , severe redness and swelling of the entire paw including digits; 4, maximally inflamed limb with involvement of multiple joints.

\section{X-ray micro-tomography}

After blood sampling, all 4 limbs were dissected and fixed in $4 \%$ paraformaldehyde ( $\mathrm{pH}$ 7.4). The tibias were subjected to three-dimensional micro-computed tomography. Three-dimensional microstructural image data obtained were reconstructed using TRI/3D-BON software (Ratoc System Engineering Co., Ltd., Tokyo, Japan).

\section{Histological examinations}

The fixed limbs were decalcified in a decalcifying solution from Wako Pure Chemicals Co., Ltd. (Osaka, Japan) and embedded in paraffin. They were finally sliced into $8-\mu \mathrm{m}$ sections, stained with hematoxylin and eosin, and observed under a microscope. For observation of the articular cartilage, the sections were stained with $0.1 \%$ Fast Green for 5 minutes, rinsed with $1 \%$ acetic acid for 10 seconds, and stained with Safranin O for 5 minutes.

\section{Statistical analyses}

Results are expressed as the mean \pm SD. A MannWhitney $U$ test and Steel-Dwass test were used for statistical analyses, with $P$ values less than 0.05 considered to be significant.

\section{Additional file}

Additional file 1: Figure S1. Effect of anti-RANKL antibody on change in the thickness of footpads in mice with or without injection of a cocktail of anti-type II collagen antibodies. DBA1/J mice in the RA+ groups were given an intra-peritoneal injection of a cocktail of 5 clones of mouse monoclonal anti-type II collagen antibodies (Chondrex) on day 0 , followed by an intra-peritoneal injection of E. coli LPS on day 3. RA- mice were the control without injection of the anti-type II collagen antibodies and LPS. The OYC1 anti-RANKL monoclonal antibody was injected subcutaneously into mice in the Ab+ groups on day 5 , while mice in the Ab- groups were not given that treatment. The thickness of each footpad was measured daily from day 0 to day 14 . Change in the thickness of each footpad was plotted as a line for individual mice. The left panels show the results from RA- mice $(A, C, E, G)$, and the right panels are those from $R A+$ mice $(B, D, F, H)$. Dotted and solid lines indicate the values obtained in $A b$ - and $A b+$ mice, respectively. Results from left front paws $(A, B)$, right front paws $(C, D)$, left hind paws $(E, F)$, and right hind paws $(G, H)$ were indicated as separate figures.

\section{Abbreviations}

RA: Rheumatoid arthritis; RANK: Receptor activator of nuclear factor $\mathrm{k}-\mathrm{B}$; RANKL: RANK ligand; LPS: Lipopolysaccharide; BV/TV: Bone volume fraction; OPG: Osteoprotegerin.

\section{Competing interests}

The authors declare that they have no competing interests.

\section{Authors' contributions}

SF and AM performed the major parts of the animal experiments including induction of arthritis, administration of the anti-RANK antibody, and scoring inflammatory response in limbs. KO, YM, and KY planned the study and prepared the manuscript. JT, HS, KM, and DS performed histological analyses. $\mathrm{RU}, \mathrm{MN}, \mathrm{ON}, \mathrm{KB}, \mathrm{Kl}$, and RK provided expert advice for performing the experiments and preparing the manuscript. All authors have seen and approved the final version of the manuscript prior to submission.

\section{Acknowledgements}

This study was supported in part by Grants-in-Aid for Scientific Research from the Japan Society for the Promotion of Sciences, and grants from the Project to Establish Strategic Research Center for Innovative Dentistry established by The Ministry of Education, Culture, Sports, Science and Technology of Japan.

\section{Author details}

${ }^{1}$ Department of Biochemistry, Showa University School of Dentistry, 1-5-8 Hatanodai, Shinagawa, Tokyo 142-8555, Japan. ${ }^{2}$ Department of Orthopaedic Surgery, Showa University School of Medicine, Tokyo, Japan. ${ }^{3}$ Department of Orthopaedic Surgery, Showa University Northern Yokohama Hospital,

Yokohama, Japan. ${ }^{4}$ Department of Prosthodontics, Showa University School of Dentistry, Tokyo, Japan. ${ }^{5}$ Department of Oral Diagnostic Sciences, Division of Pathology, Showa University School of Dentistry, Tokyo, Japan.

Received: 30 August 2014 Accepted: 20 November 2014

Published online: 12 December 2014

\section{References}

1. Ospelt C, Neidhart M, Gay RE, Gay S: Synovial activation in rheumatoid arthritis. Front Biosci 2004, 9:2323-2334.

2. Tanaka S, Nakamura K, Takahashi N, Suda T: Role of RANKL in physiological and pathological bone resorption and therapeutics targeting the RANKLRANK signaling system. Immunol Rev 2005, 208:30-49.

3. Nakashima T, Hayashi M, Fukunaga T, Kurata K, Oh-Hora M, Fung JQ, Bonewald LF, Kodama T, Wutz A, Wagner EF, Penninger JM, Takayanagi H: Evidence for osteocyte regulation of bone homeostasis through RANKL expression. Nat Med 2011, 17:1231-1234.

4. Goldring SR: Pathogenesis of bone and cartilage destruction in rheumatoid arthritis. Rheumatology 2003, 42(Suppl):ii1-ii16.

5. Takayanagi $\mathrm{H}$ : New developments in osteoimmunology. Nat Rev Rheumatol 2012, 8:684-689.

6. Cummings SR, San Martin J, McClung MR, Siris ES, Eastell R, Reid IR, Delmas P, Zoog HB, Austin M, Wang A, Kutiluk S, Adami S, Zanchetta J, Libanati C, Siddhanti S, Christansen C, Freedom Trial: Denosumab for prevention of fractures in postmenopausal women with osteoporosis. N Engl J Med 2009, 361:756-765.

7. Yee AJ, Raje NS: Denosumab, a RANK kigand inhibitor, for the management of bone loss in cancer patients. Clin Interv Aging 2012, 7:331-338.

8. Cohen SB, Dore RK, Lane NE, Ory PA, Peterfy CG, Sharp JT, van der Heijde D, Zhou L, Tsuji W, Newmark R: Denosumab treatment effects on structural 
damage, bone mineral density, and bone turnover in rheumatoid arthritis. Arthritis Rheumat 2008, 58:1299-1309.

9. Sinningen K, Tsourdi E, Rauner M, Rachner TD, Hamann C, Hofbauer LC: Skeletal and extraskeletal actions of denosumab. Endocrine 2012, 42:52-62.

10. Li Z, Hou W-S, Brömme D: Collagenolytic activity of cathepsin K is specifically modulated by cartilage-resident chondroitin sulfates. Biochemistry 2000, 39:529-536.

11. Hou W-S, Li W, Keyszer G, Weber E, Levy R, Klein MJ, Gravallese EM, Goldring $\mathrm{SR}$, Brömme D: Comparison of cathepsin $\mathrm{K}$ and $\mathrm{S}$ expression within the rheumatoid and osteoarthritic synovium. Arthritis Rheum 2002, 46:663-674.

12. Svelander L, Erlandsson-Harris H, Astner L, Grabowska U, Klareskog L, Lindstrom $\mathrm{E}$, Hewitt $\mathrm{E}$ : Inhibition of cathepsin $\mathrm{K}$ reduces bone erosion, cartilage degradation and inflammation evoked by collagen-induced arthritis in mice. Eur J Pharmacol 2009, 613:155-162.

13. Furuya Y, Mori K, Ninomiya T, Tomimori Y, Yanaka S, Takahashi N, Udagawa $\mathrm{N}$, Uchida K, Yasuda H: Increased bone mass in mice after single injection of anti-receptor activator of nuclear factor-kB ligand-neutralizing antibody: evidence for bone anabolic effect of parathyroid hormone in mice with few osteoclasts. J Biol Chem 2011, 286:37023-37031.

14. Leibbrandt $A$, Penninger JM: RANK/RANKL: regulators of immune responses and bone physiology. Ann NY Acad Sci 2008, 1143:123-150.

15. Lacey DL, Timms E, Tan HL, Kelley MJ, Dunstan CR, Burgess T, Elliott R, Colombero A, Elliott G, Scully S, Hsu H, Sullivan J, Hawkins N, Davy E, Capparelli C, Eli A, Qian YX, Kaufman S, Sarosi I, Shalhoub V, Senaldi G, Guo J, Delaney J, Boyle WJ: Osteoprotegerin ligand is a cytokine that regulates osteoclast differentiation and activation. Cell 1998, 93:165-176.

16. Yasuda H, Shima N, Nakagawa N, Yamaguchi K, Kinosaki M, Mochizuki S, Tomoyasu A, Yano K, Goto M, Murakami A, Tsuda E, Morinaga T, Higashio K, Udagawa N, Takahashi N, Suda T: Osteoclast differentiation factor is a ligand for osteoprotegerin/osteoclastogenesis-inhibitory factor and is identical to TRANCE/RANKL. Proc Natl Acad Sci U S A 1998, 95:3597-3602.

17. Romas E, Sims NA, Hards DK, Lindsay M, Quinn JWM, Ryan PFJ, Dunstan CR, Martin J, Gillespie MT: Osteoprtegerin reduces osteoclast numbers and prevents bone erosion in collagen-induced arthritis. Am J Pathol 2002, 161:1419-1427.

18. Redlich K, Görtz B, Hayer S, Zwerina J, Doerr N, Kostenuik P, Bergmeister H, Kollias G, Steiner G, Snolen JS, Schett G: Repair of local bone erosions and reversal of systemic bone loss upon therapy with anti-tumor necrosis factor in combination with osteoprotegerin or parathyroid hormone in tumor necrosis factor-mediated arthritis. Am J Pathol 2004, 164:543-555.

19. Schett G, Middleton S, Bolon B, Stolina M, Brown H, Zhu L, Pretorius J, Zak $D J$, Kostenuik P, Feige U: Additive bone-protective effects of anabolic treatment when used in conjunction with RANKL and tumor necrosis factor inhibition in two rat arthritis models. Arthritis Rheumat 2005, $52: 1604-1611$

20. Stolina M, Schett G, Dwyer D, Vonderfecht S, Middleton S, Duryea D, Pacheco E, Van G, Bolon B, Feige U, Zack D, Kostenuil P: RANKL inhibition by osteoprotegerin prevents bone loss without affecting local or systemic inflammation parameters in two rat arthritis models: comparison with anti-TNFa or anti-IL-1 therapies. Arthritis Res Ther 2009, 11:1-15.

doi:10.1186/s12952-014-0018-0

Cite this article as: Funato et al:: Effects of antibody to receptor activator of nuclear factor $\mathrm{K}-\mathrm{B}$ ligand on inflammation and cartilage degradation in collagen antibody-induced arthritis in mice. Journal of Negative Results in BioMedicine 2014 13:18.

\section{Submit your next manuscript to BioMed Central and take full advantage of:}

- Convenient online submission

- Thorough peer review

- No space constraints or color figure charges

- Immediate publication on acceptance

- Inclusion in PubMed, CAS, Scopus and Google Scholar

- Research which is freely available for redistribution 